\title{
Hispanic or Latino
}

National Cancer Institute

\section{Source}

National Cancer Institute. Hispanic or Latino. NCI Thesaurus. Code C17459.

A person of Cuban, Mexican, Puerto Rican, South or Central American, or other Spanish culture or origin, regardless of race. The term, "Spanish origin," can be used in addition to "Hispanic or Latino." (OMB) 